\title{
Improved Lower Bounds for Ranging in Synchronous Visible Light Positioning Systems
}

\author{
Musa Furkan Keskin, Erdal Gonendik, and Sinan Gezici, Senior Member, IEEE
}

\begin{abstract}
In this study, the Ziv-Zakai bound (ZZB) is derived for synchronous visible light positioning (VLP) systems. The proposed ZZB extracts ranging information from the prior information, the time delay parameter, and the channel attenuation factor based on the Lambertian pattern. In addition to the $\mathrm{ZZB}$, the Bayesian Cramér-Rao bound (CRB) and the weighted CRB (WCRB) are calculated for synchronous VLP systems. Furthermore, a closed-form expression is obtained for the expectation of the conditional CRB (ECRB). Numerical examples are presented to compare the bounds against each other and against the maximum a posteriori probability (MAP) estimator. It is observed that the ZZB can provide a reasonable lower limit on the performance of MAP estimators. On the other hand, the WCRB and the ECRB converge to the $\mathrm{ZZB}$ in regions of low and high source optical powers, respectively; however, they are not tight in other regions.
\end{abstract}

Index Terms-Estimation, Lambertian pattern, positioning, visible light, Ziv-Zakai bound.

\section{INTRODUCTION}

I N INDOOR environments, light emitting diode (LED) based visible light systems can be used for accurate positioning, high speed data transmission, and illumination simultaneously [1]-[9]. The topic of this manuscript is related to positioning via visible light systems. Visible light positioning (VLP) systems with high localization accuracy can be employed in numerous applications including robot navigation and asset tracking [4], [10]. Recently, various studies have been performed on VLP systems, and high positioning accuracies have been reported based on experiments and simulations; e.g., [11]-[13]. The aim in this manuscript is to obtain theoretical limits on distance (range) estimation in a synchronous VLP system, which provide performance benchmarks for practical estimators.

Depending on the presence/absence of synchronization among LED transmitters and visible light communication (VLC) receivers, VLP systems can be categorized as synchronous and asynchronous. In an asynchronous VLP system, LED transmitters are not synchronized with VLC receivers, and the main parameter utilized for positioning is the received signal strength (RSS) (or, power) of the incoming signal based on the Lambertian formula [7], [8], [11], [13]-[15]. On the other hand,

Manuscript received January 25, 2016; revised July 19, 2016 and September 20, 2016; accepted October 19, 2016. Date of publication October 23, 2016; date of current version November 17, 2016. This work was supported in part by the Distinguished Young Scientist Award of Turkish Academy of Sciences (TUBA-GEBIP 2013).

The authors are with the Department of Electrical and Electronics Engineering, Bilkent University, 06800 Ankara, Turkey (e-mail: keskin@ee.bilkent. edu.tr; erdal@ee.bilkent.edu.tr; gezici@ee.bilkent.edu.tr).

Color versions of one or more of the figures in this paper are available online at http://ieeexplore.iee.org.

Digital Object Identifier 10.1109/JLT.2016.2620518 in a synchronous VLP system, where LED transmitters and VLC receivers are synchronized, both the time-of-arrival (TOA) and the RSS parameters can be utilized for distance (hence, position) estimation [5], [16]. Also, in the presence of multiple photo-detectors at the VLC receiver, the angle-of-arrival (AOA) parameter can also be employed for positioning [9], [17], [18]. The AOA can be calculated from the TOA differences or the RSS differences among the photo-detectors depending on the presence/absence of synchronization.

Theoretical accuracy limits are important for VLP systems to present benchmarks for numerous studies in the literature. In [5], [6], [16], [19], the Cramér-Rao bound (CRB) is considered for providing theoretical limits for various VLP systems. In [6], the CRB on range (distance) estimation is derived for an asynchronous VLP system based on RSS measurements, and the effects of system parameters, such as the signal bandwidth, LED configuration and transmitter height, are investigated. The study in [5] focuses on a synchronous VLP system and presents the CRB on TOA based range estimation. It also analyzes the impact of various system parameters, such as the area of the photo detector, source optical power, and center frequency, on ranging accuracy. In [16], the CRBs and maximum likelihood estimators (MLEs) are investigated for both synchronous and asynchronous VLP systems. In the synchronous case, both the channel attenuation factor (RSS) and the TOA parameters are utilized while only the RSS parameter is used in the asynchronous case. Comparisons are performed among the synchronous and asynchronous scenarios based on the analytical CRB expressions [16]. In [19], a hybrid AOA and RSS based three-dimensional localization is investigated for an asynchronous VLP system, where AOA based and RSS based localization algorithms employ, respectively, a least-squares estimator and an analytical learning rule based on the NewtonRaphson method. Also, the CRB is derived for RSS based three dimensional localization for a generic deployment scenario. Unlike the theoretical limits in [5], [6], [16], [19], the aim in this study is to provide theoretical limits for a synchronous VLP system by considering the effects of prior information, as well.

Although the CRB can provide tight limits on mean-squared errors (MSEs) of unbiased estimators in high signal-to-noise ratio (SNR) conditions, it can be quite loose for low SNRs [20]. In addition, the CRB derivations do not consider any prior statistical information about the range (or, position) parameter, which can in fact be available in indoor environments; e.g., based on physical dimensions and known system parameters such as the field of view of the photo detector. To address these issues, the Ziv-Zakai bound (ZZB) can be used as a benchmark 
for ranging in VLP systems. The ZZB can provide tight limits on MSEs of estimators in all SNR conditions, and it also utilizes the available prior information [20], [21]. The study in [22] derives the ZZB on range estimation in an asynchronous VLP system based on RSS measurements and provides comparisons with the maximum a-posteriori probability (MAP) and the minimum mean-squared error (MMSE) estimators.

In this paper, the $\mathrm{ZZB}$ on ranging is derived for a synchronous VLP system by utilizing the prior information and the ranging information from both the time delay (TOA) parameter and the channel attenuation factor (RSS) via the Lambertian pattern. Based on the ZZB, effects of various system parameters, such as the Lambertian order, the area of the photo detector, and the source optical power, are analyzed in terms of ranging accuracy, and design guidelines are provided for practical VLP systems. In addition, the expectation of the CRB (ECRB) is calculated and a closed-form expression is obtained for uniform prior information. The ECRB expression both illustrates the effects of prior information and provides a low-complexity alternative to the ZZB in high SNR conditions. Moreover, the Bayesian CRB (BCRB) and the weighted CRB (WCRB) are derived in order to present theoretical limits that effectively utilize the prior information, and they are compared against the ZZB. The main contributions of this study can be summarized as follows:

1) The $Z Z B$ on ranging is derived for a synchronous VLC system by utilizing prior information together with the ranging information extracted from the time delay parameter and the channel attenuation factor. (The provided ZZB expression is different from those for synchronous RF systems [20], [23], [24] due to the facts that (i) synchronous VLP systems utilize both time delay and received signal power information whereas synchronous RF systems use time delay information only, and (ii) the Lambertian formula is available for VLP systems to specify the received signal power, which is not valid for RF systems.)

2) A closed-form ECRB expression is derived for ranging in synchronous VLC systems, which converges to the ZZB in the high SNR regime.

3) The BCRB and the WCRB expressions are provided for a synchronous VLC system, which have not been available in the literature.

4) Performance of the MAP estimator is compared against the theoretical limits. It is demonstrated that the theoretical limits on the performance of the MAP estimators can be characterized by the $\mathrm{ZZB}$, which provides important guidelines for designers of practical VLP systems. In addition, the ECRB and the WCRB are observed to converge to the ZZB in the high and low SNR regimes, respectively.

The rest of the paper is organized as follows: The system model is introduced in Section II. The ZZB for synchronous VLP systems is derived in Section III, and a closed-form ECRB expression is provided in Section IV. The BCRB and the WCRB expressions are obtained in Section V. Numerical results investigating the performance of the MAP estimator and the bounds are presented in Section VI, followed by some concluding remarks in Section VII.

\section{SYSTEM MODEL}

Consider an LED transmitter and a VLC receiver that are located at a distance of $x$ from each other. A line-of-sight (LOS) scenario is assumed, which is commonly the case for visible light systems [4], [5]. Then, the received signal at the VLC receiver is stated as [5]

$$
r(t)=\alpha R_{p} s(t-\tau)+n(t)
$$

for $t \in\left[T_{1}, T_{2}\right]$, where $T_{1}$ and $T_{2}$ determine the observation interval, $\alpha$ is the attenuation factor of the optical channel $(\alpha>0)$, $R_{p}$ is the responsivity of the photo detector, $s(t)$ is the transmitted signal which is nonzero over an interval of $\left[0, T_{s}\right], \tau$ is the time-of-arrival (TOA), and $n(t)$ is zero-mean additive white Gaussian noise with spectral density level $\sigma^{2}$. Considering a synchronous system as in [5], the TOA parameter is modeled as

$$
\tau=\frac{x}{c}
$$

where $x$ is the distance (range) between the LED transmitter and the VLC receiver, and $c$ is the speed of light. It is assumed that the signal component in (1) is contained completely in the observation interval $\left[T_{1}, T_{2}\right]$; that is, $\tau \in\left[T_{1}, T_{2}-T_{s}\right]$. In (1), the channel attenuation factor $\alpha$ is modeled as

$$
\alpha=\frac{m+1}{2 \pi} \cos ^{m}(\phi) \cos (\theta) \frac{S}{x^{2}}
$$

where $m$ is the Lambertian order, $\phi$ is the irradiation angle, $\theta$ is the incidence angle, and $S$ is the area of the photo detector at the VLC receiver [5]. For clarity of theoretical expressions, it is assumed, as in [5], [6], [11], [22], that the LED transmitter is pointing downwards (which is commonly the case since LEDs are employed also for illumination) and the photo detector at the VLC receiver is pointing upwards. Then, $\phi=\theta$ and $\cos (\phi)=\cos (\theta)=h / x$, where $h$ denotes the height of the LED transmitter relative to the VLC receiver. (The theoretical expressions in this study can also be extended to the cases with arbitrary transmitter and receiver orientations, which however leads to lengthy and inconvenient expressions.) In addition, as in [5], [6], [8], [11], [22], it is assumed that the LED transmitter is at a known height with respect to the VLC receiver; i.e., possible locations of the VLC receiver are confined to a two-dimensional plane. This assumption is valid in various practical applications; e.g., when the VLC receiver is attached to a cart or a robot that is tracked via a VLP system since VLC receivers have fixed and known heights in those applications (e.g., [4, Fig. 3]). Under these assumptions, (3) reduces to

$$
\alpha=\frac{m+1}{2 \pi}\left(\frac{h}{x}\right)^{m+1} \frac{S}{x^{2}} \triangleq \gamma x^{-m-3}
$$

where $\gamma$ is a known constant defined as

$$
\gamma \triangleq \frac{(m+1) h^{m+1} S}{2 \pi} .
$$

\section{ZIV-ZAKAI BOUND (ZZB)}

The ZZB provides a lower limit on MSEs of estimators based on a relation in terms of the probability of error in a binary 
hypothesis-testing problem. It is expressed as [21]

$$
\xi \geq \frac{1}{2} \int_{0}^{\infty} \int_{-\infty}^{\infty}(w(\vartheta)+w(\vartheta+\delta)) \mathrm{P}_{\min }(\vartheta, \vartheta+\delta) d \vartheta \delta d \delta
$$

where $\xi=\mathbb{E}\left\{|\hat{x}-x|^{2}\right\}$ is the MSE of an estimator $\hat{x}, w(\cdot)$ represents the prior probability density function (PDF) of parameter $x$, and $\mathrm{P}_{\min }(\vartheta, \vartheta+\delta)$ denotes the probability of error corresponding to the optimal decision rule for the following hypothesis-testing problem:

$$
\begin{aligned}
& \mathcal{H}_{0}: p(r(t) \mid x=\vartheta) \\
& \mathcal{H}_{1}: p(r(t) \mid x=\vartheta+\delta)
\end{aligned}
$$

In practical indoor scenarios, lower and upper limits on the range parameter $x$ are available based on physical dimensions of the environment and the field of view of the photo detector. Hence, it is reasonable to assume that the prior PDF of $x$ is zero outside the interval $\left[D_{1}, D_{2}\right]$, where $D_{1}$ and $D_{2}$ denote the minimum and maximum possible distances, respectively. (For the signal model in (1), the observation interval $\left[T_{1}, T_{2}\right]$ can be related to $D_{1}$ and $D_{2}$ as $T_{1}=D_{1} / c$ and $T_{2}=D_{2} / c+T_{s}$.) In this case, the ZZB in (6) is expressed as

$$
\begin{aligned}
\xi \geq & \frac{1}{2} \int_{0}^{D_{2}-D_{1}} \int_{D_{1}}^{D_{2}-\delta}(w(\vartheta)+w(\vartheta+\delta)) \\
& \times \mathrm{P}_{\min }(\vartheta, \vartheta+\delta) d \vartheta \delta d \delta .
\end{aligned}
$$

For example, if the prior PDF of $x$ corresponds to uniform distribution over $\left[D_{1}, D_{2}\right]$ (that is, $w(x)=1 /\left(D_{2}-D_{1}\right)$ if $x \in$ $\left[D_{1}, D_{2}\right]$ and $w(x)=0$ otherwise), the ZZB in (8) reduces to

$$
\xi \geq \frac{1}{D_{2}-D_{1}} \int_{0}^{D_{2}-D_{1}} \int_{D_{1}}^{D_{2}-\delta} \mathrm{P}_{\min }(\vartheta, \vartheta+\delta) d \vartheta \delta d \delta .
$$

To obtain an explicit expression for the $\mathrm{ZZB}, \mathrm{P}_{\min }(\vartheta, \vartheta+\delta)$ in (8) should be specified. Based on the PDF $w(x)$ of $x$, the prior probabilities of hypotheses $\mathcal{H}_{0}$ and $\mathcal{H}_{1}$ in (7) are equal to $w(\vartheta) /(w(\vartheta)+w(\vartheta+\delta))$ and $w(\vartheta+\delta) /(w(\vartheta)+w(\vartheta+\delta))$, respectively. Then, the optimal decision rule for the problem in (7) is the MAP rule [25], which is expressed as

$$
\begin{gathered}
\frac{w(\vartheta+\delta)}{w(\vartheta)+w(\vartheta+\delta)} p(r(t) \mid x=\vartheta+\delta) \\
\stackrel{\mathcal{H}_{0}}{\mathcal{H}_{1}} \frac{w(\vartheta)}{w(\vartheta)+w(\vartheta+\delta)} p(r(t) \mid x=\vartheta) .
\end{gathered}
$$

After taking the natural logarithm of both sides, (10) becomes

$$
\begin{aligned}
- & \log p(r(t) \mid x=\vartheta)+\log p(r(t) \mid x=\vartheta+\delta) \\
& \stackrel{\mathcal{H}_{0}}{\equiv} \log \left(\frac{w(\vartheta)}{w(\vartheta+\delta)}\right) .
\end{aligned}
$$

From (1), (2), and (4), the log-likelihood function is expressed as [26]

$\log p(r(t) \mid x)=k-\frac{1}{2 \sigma^{2}} \int_{T_{1}}^{T_{2}}\left(r(t)-\frac{R_{p} \gamma}{x^{m+3}} s\left(t-\frac{x}{c}\right)\right)^{2} d t$ where $k$ is a constant that does not depend on $x$. From (12), the decision rule in (11) be stated as

$$
\begin{aligned}
& \int_{T_{1}}^{T_{2}}\left(r(t)-\gamma \vartheta^{-m-3} R_{p} s\left(t-\frac{\vartheta}{c}\right)\right)^{2} d t \\
& -\int_{T_{1}}^{T_{2}}\left(r(t)-\gamma(\vartheta+\delta)^{-m-3} R_{p} s\left(t-\frac{\vartheta+\delta}{c}\right)\right)^{2} d t \\
& \quad \sum_{\mathcal{H}_{1}}^{\mathcal{H}_{0}} 2 \sigma^{2} \log \left(\frac{w(\vartheta)}{w(\vartheta+\delta)}\right)
\end{aligned}
$$

which reduces, via some rearrangement, to

$$
\begin{aligned}
& \frac{C_{r s}(\vartheta+\delta)}{(\vartheta+\delta)^{m+3}}-\frac{C_{r s}(\vartheta)}{\vartheta^{m+3}} \underset{\mathcal{H}_{1}}{\stackrel{\mathcal{H}_{0}}{\lesseqgtr}} \frac{R_{p} \gamma E_{s}}{2}\left(\frac{1}{(\vartheta+\delta)^{2 m+6}}\right. \\
& \left.\quad-\frac{1}{\vartheta^{2 m+6}}\right)+\frac{\sigma^{2}}{R_{p} \gamma} \log \left(\frac{w(\vartheta)}{w(\vartheta+\delta)}\right)
\end{aligned}
$$

where

$$
C_{r s}(x) \triangleq \int_{T_{1}}^{T_{2}} r(t) s\left(t-\frac{x}{c}\right) d t
$$

and

$$
E_{s} \triangleq \int_{-\infty}^{\infty}(s(t))^{2} d t
$$

is the energy of signal $s(t)$.

The probability of error for the decision rule in (14) is calculated as

$$
\begin{aligned}
\mathrm{P}_{\min }(\vartheta, \vartheta+\delta)= & \frac{w(\vartheta)}{w(\vartheta)+w(\vartheta+\delta)} \mathrm{P}\left(\hat{\mathcal{H}}_{1} \mid \mathcal{H}_{0}\right) \\
& +\frac{w(\vartheta+\delta)}{w(\vartheta)+w(\vartheta+\delta)} \mathrm{P}\left(\hat{\mathcal{H}}_{0} \mid \mathcal{H}_{1}\right)
\end{aligned}
$$

where $\mathrm{P}\left(\hat{\mathcal{H}}_{i} \mid \mathcal{H}_{j}\right)$ denotes the probability of deciding for hypothesis $\mathcal{H}_{i}$ when $\mathcal{H}_{j}$ is true. Under $\mathcal{H}_{0}$, it can be shown from (1), (4), (7), and (15) that $C_{r s}(\vartheta)$ and $C_{r s}(\vartheta+\delta)$ are jointly Gaussian distributed as

$$
\left[\begin{array}{c}
C_{r s}(\vartheta) \\
C_{r s}(\vartheta+\delta)
\end{array}\right] \sim \mathcal{N}\left(\left[\begin{array}{c}
\frac{\gamma R_{p} E_{s}}{\vartheta^{m+3}} \\
\frac{\gamma R_{p} E_{s} \rho\left(\frac{\delta}{c}\right)}{\vartheta^{m+3}}
\end{array}\right],\left[\begin{array}{cc}
\sigma^{2} E_{s} & \sigma_{s}^{2 E} \rho\left(\frac{\delta}{c}\right) \\
\sigma_{s}^{2 E} \rho\left(\frac{\delta}{c}\right) & \sigma_{s}^{2 E}
\end{array}\right]\right)
$$

where $\mathcal{N}(\boldsymbol{\mu}, \boldsymbol{\Sigma})$ represents Gaussian distribution with mean $\boldsymbol{\mu}$ and covariance matrix $\Sigma$, and $\rho(\cdot)$ represents the normalized auto-correlation function of $s(t)$, which is defined as

$$
\rho(\tau) \triangleq \frac{1}{E_{s}} \int_{-\infty}^{\infty} s(t) s(t-\tau) d t
$$

From (14) and (18), $\mathrm{P}\left(\hat{\mathcal{H}}_{1} \mid \mathcal{H}_{0}\right)$ can be calculated as

$\mathrm{P}\left(\hat{\mathcal{H}}_{1} \mid \mathcal{H}_{0}\right)=$

$Q\left(\frac{0.5 R_{p} \gamma E_{s} g(\vartheta, \vartheta+\delta)+\frac{\sigma^{2}}{\gamma R_{p}} \log (w(\vartheta) / w(\vartheta+\delta))}{\sqrt{\sigma^{2} E_{s} g(\vartheta, \vartheta+\delta)}}\right)$ 
where $Q(y)=\frac{1}{\sqrt{2 \pi}} \int_{y}^{\infty} \mathrm{e}^{-t^{2} / 2} d t$ denotes the $Q$-function, and

$$
g(\vartheta, \vartheta+\delta) \triangleq \frac{1}{(\vartheta+\delta)^{2 m+6}}+\frac{1}{\vartheta^{2 m+6}}-\frac{2 \rho(\delta / c)}{(\vartheta(\vartheta+\delta))^{m+3}}
$$

Also, via similar derivations, $\mathrm{P}\left(\hat{\mathcal{H}}_{0} \mid \mathcal{H}_{1}\right)$ can be obtained as follows:

$$
\begin{aligned}
& \mathrm{P}\left(\hat{\mathcal{H}}_{0} \mid \mathcal{H}_{1}\right)= \\
& Q\left(\frac{0.5 R_{p} \gamma E_{s} g(\vartheta, \vartheta+\delta)-\frac{\sigma^{2}}{\gamma R_{p}} \log (w(\vartheta) / w(\vartheta+\delta))}{\sqrt{\sigma^{2} E_{s} g(\vartheta, \vartheta+\delta)}}\right) .
\end{aligned}
$$

Then, the probability of error for the decision rule in (14) can be evaluated via (17), (20), and (22), which can be expressed in a compact form as follows:

$$
\mathrm{P}_{\min }(\vartheta, \vartheta+\delta)=\frac{\sum_{i=0}^{1} w(\vartheta+i \delta) \mathrm{P}\left(\hat{\mathcal{H}}_{1-i} \mid \mathcal{H}_{i}\right)}{w(\vartheta)+w(\vartheta+\delta)} .
$$

Based on the obtained minimum probability of error expression in (23), the ZZB in (8) can be calculated.

As a special case, when the prior PDF of $x$ is uniform over $\left[D_{1}, D_{2}\right]$, the logarithm terms in (20) and (22) become zero, and $\mathrm{P}_{\min }(\vartheta, \vartheta+\delta)$ in (23) can be simplified as follows:

$$
\begin{aligned}
\mathrm{P}_{\min }(\vartheta, \vartheta+\delta) & =0.5 \mathrm{P}\left(\hat{\mathcal{H}}_{1} \mid \mathcal{H}_{0}\right)+0.5 \mathrm{P}\left(\hat{\mathcal{H}}_{0} \mid \mathcal{H}_{1}\right) \\
& =Q\left(\frac{R_{p} \gamma \sqrt{E_{s}} \sqrt{g(\vartheta, \vartheta+\delta)}}{2 \sigma}\right) .
\end{aligned}
$$

For the uniform prior case, the ZZB can be calculated based on (9) and (24).

Since the integral limits in (8) and (9) are finite, the ZZB can accurately be evaluated via numerical approaches. From (8), (20), (22), and (23), it is observed that the ZZB reduces as $E_{s}$ increases; that is, improved ranging accuracy is achieved with higher transmitted signal energy, as expected. It is also noted that the ZZB expression in (8) and (23) is different from both the ZZB expression in asynchronous VLP systems [22] since the range related information from both the time delay parameter and the channel attenuation factor is employed in the synchronous case.

Remark 1: It is important to emphasize that the ZZB expression in (8) and (23) has important distinctions compared to the ZZB expressions for synchronous RF based ranging systems (e.g., [20]) due to the facts that (i) the synchronous VLP system utilizes both time delay and received signal power (channel attenuation factor) information whereas synchronous RF systems use time delay information only (since the received power parameter carries negligible information compared to the time delay parameter in most practical RF localization systems), and (ii) the Lambertian equation in (4) is available for VLP systems to relate the channel attenuation factor (the received signal power) to distance $x$ in LOS visible light channels, which is not valid for RF systems. Overall, the Lambertian formula is utilized, together with the time delay information and the prior information, for the purpose of range estimation in this study.

\section{ECRB DERIVATIONS}

In this section, the Cramér-Rao bound (CRB) expressions for range estimation in VLP systems are investigated to provide comparisons against the ZZB.

For a given value of the unknown parameter, the conditional $C R B$ presents a lower limit on the MSEs of unbiased estimators, which is expressed as [21]

$$
\begin{aligned}
\mathbb{E}\left\{|\hat{x}-x|^{2}\right\} & \geq\left(\mathbb{E}\left\{\left(\frac{\partial \log p(r(t) \mid x)}{\partial x}\right)^{2}\right\}\right)^{-1} \\
& \triangleq\left(\mathrm{J}_{\mathrm{F}}(x)\right)^{-1}=\mathrm{CRB}(x)
\end{aligned}
$$

where $\hat{x}$ is an unbiased estimate of $x$ and the expectation operators are conditioned on $x$. For the estimation of the range parameter $x$, the conditional CRB in the synchronous case can be obtained from (25) as [16]

$$
\operatorname{CRB}(x)=\left(\mathrm{J}_{\mathrm{F}}(x)\right)^{-1}=\frac{\left(\sigma x^{m+4} /\left(\gamma R_{p}\right)\right)^{2}}{(m+3)^{2} E_{s}+\tilde{E}_{s}(x / c)^{2}}
$$

where $\tilde{E}_{s} \triangleq \int_{0}^{T_{s}}\left(s^{\prime}(t)\right)^{2} d t$, with $s^{\prime}(t)$ denoting the first-order derivative of $s(t) .{ }^{1}$ The conditional CRB expression in (26) is a function of the unknown parameter $x$, and no prior information is considered in the derivation of this bound.

The expectation of the conditional CRB (ECRB): is obtained by calculating the average of the conditional CRB over the prior distribution of the unknown parameter [21], which results in the following expression for the considered scenario:

$$
\operatorname{ECRB}=\mathbb{E}\{\operatorname{CRB}(x)\}=\int_{D_{1}}^{D_{2}} w(x) \operatorname{CRB}(x) d x
$$

where $\operatorname{CRB}(x)$ denotes the conditional CRB in (26), and $w(x)$ is the prior PDF of $x$, which is zero outside $\left[D_{1}, D_{2}\right]$. For the uniform prior PDF, the ECRB is specified as in the following lemma:

Lemma 1: Suppose that the prior PDF of $x$ is specified by a uniform distribution over $\left[D_{1}, D_{2}\right]$. Then, the ECRB in the synchronous case is given by

$$
\mathrm{ECRB}=\frac{\left(\sigma /\left(\gamma R_{p}\right)\right)^{2}}{\left(D_{2}-D_{1}\right) \tilde{E}_{s} / c^{2}} \int_{D_{1}}^{D_{2}} \frac{x^{2 m+8}}{x^{2}+a} d x
$$

with $a \triangleq(m+3)^{2} c^{2} E_{s} / \tilde{E}_{s}$, which can be stated as in the following expression when $2 m$ is an integer:

$$
\begin{aligned}
\operatorname{ECRB}= & \frac{\left(\sigma /\left(\gamma R_{p}\right)\right)^{2}}{\left(D_{2}-D_{1}\right) \tilde{E}_{s} / c^{2}}\left((-a)^{\lfloor m+3\rfloor+1} H_{m}\left(D_{1}, D_{2}, a\right)\right. \\
& \left.+\sum_{i=0}^{\lfloor m+3\rfloor} \frac{(-a)^{i}\left(D_{2}^{2(m+3-i)+1}-D_{1}^{2(m+3-i)+1}\right)}{2(m+3-i)+1}\right)
\end{aligned}
$$

\footnotetext{
${ }^{1}$ For the expression in (26), it is assumed that $s(0)=s\left(T_{s}\right)$, which is commonly the case [16].
} 
where

$$
\begin{aligned}
& H_{m}\left(D_{1}, D_{2}, a\right) \triangleq \\
& \begin{cases}\left(\tan ^{-1}\left(D_{2} / \sqrt{a}\right)-\tan ^{-1}\left(D_{1} / \sqrt{a}\right)\right) / \sqrt{a} & \text { if } m \in \mathbb{Z}^{+} \\
0.5\left(\ln \left(D_{2}^{2}+a\right)-\ln \left(D_{1}^{2}+a\right)\right), & \text { if } 2 m \in \mathbb{Z}^{+} m \notin \mathbb{Z}^{+}\end{cases}
\end{aligned}
$$

Proof: The generic expression in (28) directly follows from (26) and (27). To derive the specific expressions in (29) and (30), consider the division of $x^{2 m+8}$ by $x^{2}+a$, which results in the following relation:

$x^{2 m+8}=\left(x^{2}+a\right) \sum_{i=0}^{j}(-a)^{i} x^{2(m+3-i)}-(-1)^{j} a^{j+1} x^{2(m+3-j)}$

where $j \leq m+3$ is an integer. Then, the integral in (28) becomes

$$
\begin{aligned}
\int_{D_{1}}^{D_{2}} \frac{x^{2 m+8}}{x^{2}+a} d x= & \int_{D_{1}}^{D_{2}} \sum_{i=0}^{j}(-a)^{i} x^{2(m+3-i)} d x \\
& -(-1)^{j} a^{j+1} \int_{D_{1}}^{D_{2}} \frac{x^{2(m+3-j)}}{x^{2}+a} d x .
\end{aligned}
$$

If $m$ is a positive integer, $j=m+3$ can be employed to obtain the result specified by (29) and the first part of (30). (Note that the last integral term in (32) becomes $\int_{D_{1}}^{D_{2}}\left(x^{2}+a\right)^{-1} d x$ in this case, which leads to the $\tan ^{-1}$ terms in (30).) Similarly, if $m$ is not an integer but $2 m$ is a positive integer, then $j=\lfloor m+3\rfloor$ can be used to derive the expression specified by (29) and the second part of (30). (Note that the last integral term in (32) becomes $\int_{D_{1}}^{D_{2}} x\left(x^{2}+a\right)^{-1} d x$ in this case, which results in the logarithm terms in (30)).

The ECRB may not provide a lower bound on the performance of MAP estimators since the conditional CRBs, which are the basis for the ECRB as described above, do not take the prior information into account [21]. However, at high SNRs, the ECRB can converge to the $\mathrm{ZZB}$, which is expected since the prior information becomes negligible compared to the information gathered from the measurements in high SNR conditions. Overall, the ECRB provides useful benchmarks for comparisons against the ZZB and helps quantify the range related information gathered from prior information, as investigated in Section VI. In addition, the ECRB expressions provide a low-complexity approach (compared to the ZZB expressions) for calculating the theoretical limits on range estimation in high SNR scenarios.

\section{BAYESIAN CRB (BCRB) AND WEIGHTED CRB (WCRB)}

In order to incorporate the prior information into the lower bound effectively, the Bayesian CRB $(B C R B)$ can be considered [21]. The BCRB is expressed as

$$
\xi \geq\left(\mathbb{E}\left\{\left(\frac{\partial \log p(r(t) \mid x)}{\partial x}\right)^{2}\right\}+\mathbb{E}\left\{\left(\frac{\partial \log w(x)}{\partial x}\right)^{2}\right\}\right)^{-1}
$$

where $\xi=\mathbb{E}\left\{|\hat{x}-x|^{2}\right\}$ denotes the MSE of an estimator $\hat{x}$ [21]. In (33), the first expectation operator is with respect to both $r(t)$ and $x$ while the second expectation is over parameter $x$ only. From (25) and (26), the first term in (33) can be calculated as follows:

$$
\begin{aligned}
& \mathbb{E}\left\{\left(\frac{\partial \log p(r(t) \mid x)}{\partial x}\right)^{2}\right\}=\mathbb{E}\left\{\mathrm{J}_{\mathrm{F}}(x)\right\} \\
& =\int_{D_{1}}^{D_{2}} w(x) \frac{(m+3)^{2} E_{s}+\tilde{E}_{s}(x / c)^{2}}{\left(\sigma x^{m+4} /\left(\gamma R_{p}\right)\right)^{2}} d x .
\end{aligned}
$$

For a given prior PDF, the BCRB can be obtained based on (33) and (34). One of the limitations of the BCRB is due to the existence and absolute integrability requirement for the partial derivative of the joint PDF of the observation and the parameter [21]. Therefore, it may not be applicable in some scenarios. For example, when the range parameter is uniformly distributed over $\left[D_{1}, D_{2}\right]$, the BCRB does not exist.

The weighted $C R B(W C R B)$ provides an alternative to the $\mathrm{BCRB}$ and handles the existence problem. It is defined as [21]

$$
\xi \geq \frac{(\mathbb{E}\{q(x)\})^{2}}{\mathbb{E}\left\{q^{2}(x) \mathrm{J}_{\mathrm{F}}(x)\right\}+\mathbb{E}\left\{q^{2}(x)\left(\frac{d \log (w(x) q(x))}{d x}\right)^{2}\right\}}
$$

where $\xi$ is the MSE of any estimator, $\mathrm{J}_{\mathrm{F}}(x)$ is as in $(25), q(x)$ is a weighting function, and the expectations are with respect to $x$. As in [21], the following weighting function can be employed:

$$
q(x)=\left(\frac{x-D_{1}}{D_{2}-D_{1}}\right)^{\nu}\left(1-\frac{x-D_{1}}{D_{2}-D_{1}}\right)^{\nu}
$$

for $x \in\left[D_{1}, D_{2}\right]$ and $q(x)=0$ otherwise, where $\nu$ is a parameter used to enhance the bound. Namely, the value of $\nu$ that maximizes the bound in (35) is employed to obtain the tightest bound. For the uniform prior PDF, $\mathbb{E}\{q(x)\}$ in (35) is calculated from (36) as follows:

$$
\mathbb{E}\{q(x)\}=\frac{1}{D_{2}-D_{1}} \int_{D_{1}}^{D_{2}} q(x) d x=\beta(\nu+1, \nu+1)
$$

where $\beta(a, b) \triangleq \int_{0}^{1} x^{a-1}(1-x)^{b-1} d x$ denotes the beta function. In addition, the second term in the denominator of (35) can be expressed for the uniform prior PDF as [22]

$$
\mathbb{E}\left\{q^{2}(x)\left(\frac{d \log (w(x) q(x))}{d x}\right)^{2}\right\}=\frac{\nu \beta(2 \nu+1,2 \nu-1)}{\left(D_{2}-D_{1}\right)^{2}} .
$$

Also, the first term in the denominator of (35) can be calculated based on (26) and (36) as

$$
\begin{aligned}
\mathbb{E}\{ & \left.q^{2}(x) \mathrm{J}_{\mathrm{F}}(x)\right\}=\frac{\gamma^{2} R_{p}^{2} / \sigma^{2}}{\left(D_{2}-D_{1}\right)^{4 \nu+1}} \\
& \times\left((m+3)^{2} E_{s} \int_{D_{1}}^{D_{2}} \frac{\left(x-D_{1}\right)^{2 \nu}\left(D_{2}-x\right)^{2 \nu}}{x^{2 \nu+8}} d x\right. \\
& \left.+\frac{\tilde{E}_{s}}{c^{2}} \int_{D_{1}}^{D_{2}} \frac{\left(x-D_{1}\right)^{2 \nu}\left(D_{2}-x\right)^{2 \nu}}{x^{2 m+6}} d x\right) .
\end{aligned}
$$




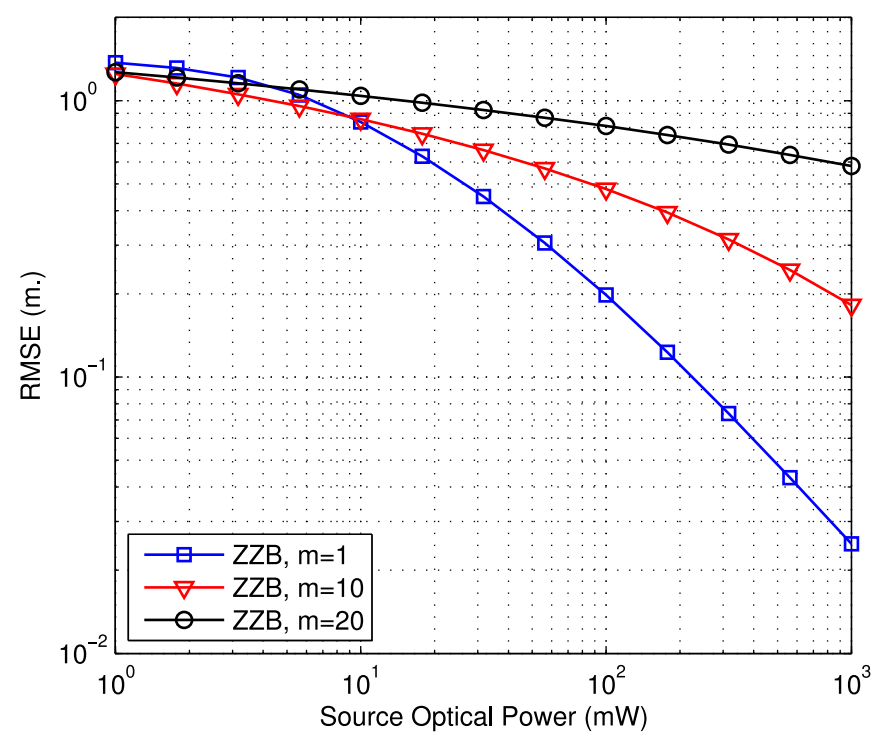

Fig. 1. ZZB versus source optical power for various values of the Lambertian order, where $S=1 \mathrm{~cm}^{2}$.

Then, the WCRB in (35) can be evaluated via (37)-(39). In order to obtain the tightest bound, the value of $\nu$ that yields the maximum lower bound is obtained.

Remark 2: The theoretical limits obtained in this study do not consider the effects of multipath (see (1)). In the presence of multipath propagation, higher MSEs would be observed in general; hence, the lower bounds for the LOS scenario provided in this manuscript present lower limits for the multipath scenario, as well. The tightness of the bounds depends on the severity of multipath effects.

\section{NUMERICAL RESULTS}

In this section, numerical examples are provided to investigate the theoretical limits. In the examples, $h$ in (4) is set to $5 \mathrm{~m}$ and the prior PDF of the distance, $x$, is taken to be uniform over the interval $\left[D_{1}, D_{2}\right]$, where $D_{1}=5 \mathrm{~m}$ and $D_{2}=10 \mathrm{~m}$ (cf. (8) and (9)). As in [5], the responsivity of the photo detector is taken as $R_{p}=0.4 \mathrm{~mA} / \mathrm{mW}$, and the spectral density level of the noise is set to $\sigma^{2}=1.336 \times 10^{-22} \mathrm{~W} / \mathrm{Hz}$. Also, signal $s(t)$ in (1) is modeled as [5]

$$
s(t)=A\left(1-\cos \left(2 \pi t / T_{s}\right)\right)\left(1+\cos \left(2 \pi f_{c} t\right)\right)
$$

for $t \in\left[0, T_{s}\right]$, where $f_{c}$ denotes the center frequency, and $A$ corresponds to the average emitted optical power; that is, source optical power.

In the first example, $T_{s}=0.1 \mathrm{~ms}, f_{c}=1 \mathrm{MHz}$, and the area $S$ of the photo detector at the VLC receiver is set to $1 \mathrm{~cm}^{2}$. In Fig. 1, the ZZBs in Section III are plotted versus the source optical power $A$ in (40) for various values of the Lambertian order $m$. It is observed that the ranging accuracy degrades as $m$ increases for practical values of the source optical power. Although the exact relation between the ZZB and $m$ can be deduced from (5), (9), and (24), an intuitive explanation can also be provided as follows: Parameter $m$ determines the directionality of the LED transmitter, and a large value of $m$ corresponds to a fast

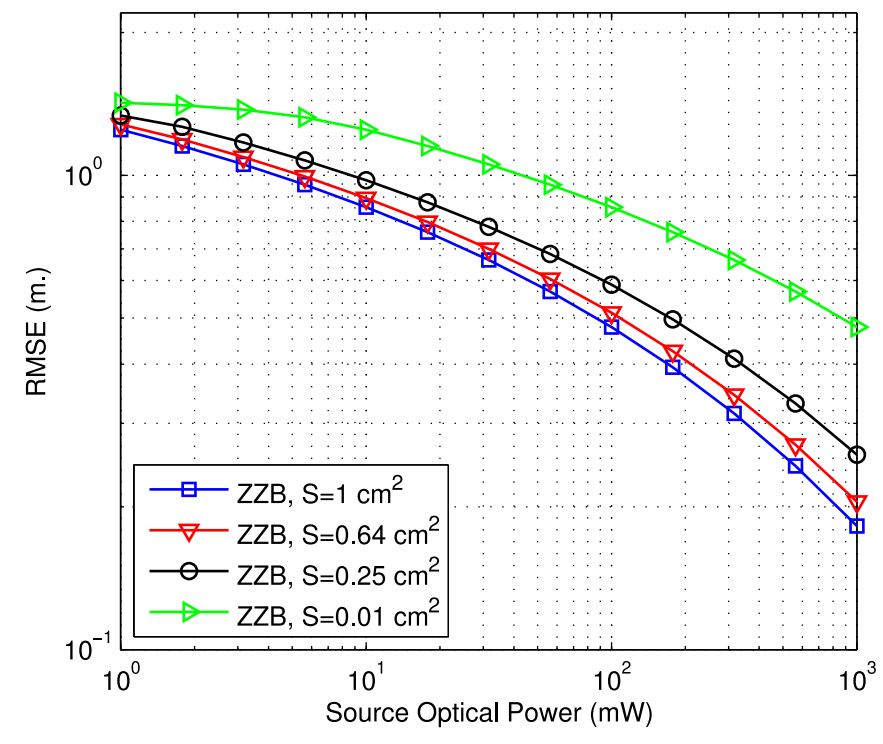

Fig. 2. ZZB versus source optical power for various values of the area of the photo detector, where $m=10$.

power decay as the irradiation angle increases from zero (see (3)). Hence, lower SNRs are expected at higher distances for larger values of $m$, which can lead to higher ZZBs, as observed in Fig. 1.

In Fig. 2, the ZZBs in Section III are presented versus the source optical power for various values of $S$, the area of the photo detector at the VLC receiver, where $T_{s}=0.1 \mathrm{~ms}, f_{c}=1 \mathrm{MHz}$, and $m=10$ are employed. From the figure, it is observed that the ZZB increases (i.e., the estimation accuracy degrades) as $S$ decreases. This observation can be explained based on the ZZB expression in (9) and (24) as follows: From (5), $\gamma$ is proportional to $S$, and from [5, eqn. (18)], $\sigma$ is proportional to $\sqrt{S}$. Hence, the $\gamma / \sigma$ term in (24) changes in proportion to $\sqrt{S}$, which leads to lower ZZBs as $S$ increases due to the monotone decreasing nature of the $Q$-function. In other words, as the area of the photo detector increases, higher SNRs are obtained at the VLC receiver and lower ZZBs are achieved.

Next, the ZZB in Section III, the ECRB in Section IV, and the WCRB in Section V are investigated in Fig. 3, together with the performance of the MAP estimator, where $T_{s}=0.1 \mathrm{~ms}$, $f_{c}=1 \mathrm{MHz}, S=1 \mathrm{~cm}^{2}$, and $m=1$. The MAP estimator can be obtained based on the ML estimator in [16, eqn. (18)] by confining the search space for the distance parameter $x$ to the interval $\left[D_{1}, D_{2}\right]$ (since the prior distribution of $x$ is uniform over $\left.\left[D_{1}, D_{2}\right]\right)$ with $D_{1}=5 \mathrm{~m}$ and $D_{2}=10 \mathrm{~m}$ Fig. 3 shows that the ECRB converges to the ZZB at high source optical powers; i.e., at high SNRs, since the prior information becomes less important as the SNR increases. However, for lower optical powers, the ECRB gets significantly higher than the ZZB since the ECRB calculations do not effectively utilize the prior information, which becomes significant in the low SNR regime. ${ }^{2}$ On

\footnotetext{
${ }^{2}$ In the ECRB calculations in (27), the prior information is used to calculate the average of the conditional CRBs; however, each conditional CRB expression is obtained without utilizing the prior information. Hence, the ECRBs do not effectively utilize the prior information.
} 


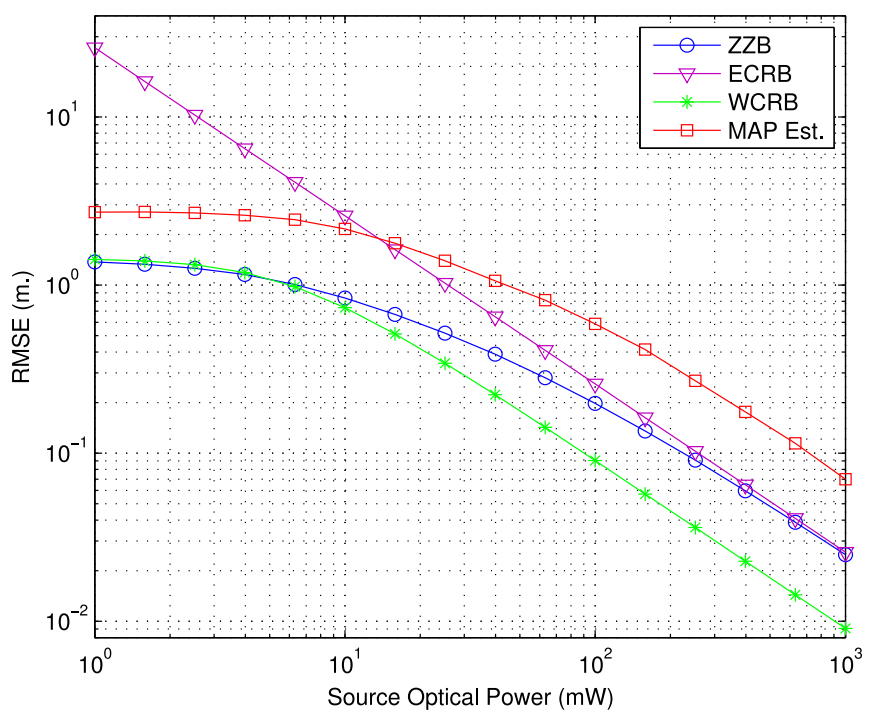

Fig. 3. RMSE versus source optical power for the MAP estimator, the ZZB, the ECRB, and the WCRB, where $T_{s}=0.1 \mathrm{~ms}, f_{c}=1 \mathrm{MHz}, S=1 \mathrm{~cm}^{2}$, and $m=1$.

the other hand, the WCRB is close to the ZZB at low SNRs but it becomes looser as the SNR increases. The main reason for this behavior is that the WCRB (and the BCRB) may not provide a tight bound at high SNRs when the conditional Fisher information depends on the unknown parameter [21, p. 7], which is the case for the considered VLP system (that is, the conditional Fisher information in (26) depends on the unknown parameter $x$, the distance between the LED transmitter and the VLC receiver). In addition, it is observed from Fig. 3 that the ZZB provides a reasonably tight bound for the performance of the MAP estimator in all SNR regions. Furthermore, since the MAP estimator utilizes the prior information, its performance cannot be lower bounded by the ECRB in the low SNR regime, which does not effectively utilize the prior information. Therefore, the ECRB expression can provide useful lower bounds only in the high SNR regime, where the prior information is not crucial in the estimation process compared to the information obtained from the received signal.

In the final example, the same parameters as in the previous scenario are employed except that a larger value of $f_{c}$ is used, namely, $f_{c}=50 \mathrm{MHz}$. The results presented in Fig. 4 illustrate that the RMSEs are reduced (i.e., the ranging performance is improved) in the medium and high SNR regimes compared to the previous scenario, which can be explained as follows: In a synchronous VLP system, in addition to the prior information, information from both the time delay parameter and the channel attenuation factor can be utilized for range estimation. Since the information gathered from the time delay parameter increases with $f_{c}$ [16], improved estimation performance can be observed at sufficiently high SNRs, where the prior information becomes less significant than the information gathered from the time delay parameter and the channel attenuation factor. However, in the low SNR regime, the prior information becomes the most significant source of information, which leads to similar performance for the MAP estimators in Figs. 3 and 4. In addition, it



Fig. 4. RMSE versus source optical power for the MAP estimator, the ZZB, the ECRB, and the WCRB, where $T_{s}=0.1 \mathrm{~ms}, f_{c}=50 \mathrm{MHz}, S=1 \mathrm{~cm}^{2}$, and $m=1$.

is noted from Fig. 4 that the MAP estimator cannot get very close to the theoretical limits at high SNRs, which is due to the finite sampling rate (namely, $10^{-11} \mathrm{~s}$ ) employed in the simulations. In particular, the finite resolution of the search for the distance parameter can introduce additional errors in the high SNR regime where the theoretical accuracy limits are quite low (see [16, eqn. (18)]). Please refer to [16, Sec. IV] for a detailed discussion.

\section{CONCLUDING REMARKS AND EXTENSIONS}

In this study, the ZZB has been derived for range estimation in synchronous VLP systems. The proposed ZZB exploits ranging information from the prior information, the time delay parameter, and the channel attenuation factor. In addition, a closed-form ECRB expression has been obtained, and the BCRB and the WCRB derivations have been presented for synchronous VLP systems. Via the numerical examples, the bounds have been compared against each other and against the MAP estimator. The ZZB has been shown to provide a reasonable lower bound for the MAP estimator. Hence, it can provide important guidelines for design of practical VLP systems. For example, based on the ZZB expression, effects of various system parameters, such as the Lambertian order, the area of the photo detector, and the source optical power, on ranging accuracy can be analyzed. On the other hand, the ECRB and the WCRB (BCRB) can provide useful bounds in the high and low SNR regimes, respectively.

For the theoretical limits in Sections III-V, the generic expressions have been presented first, and then the particular expressions have been obtained for the special case of uniform prior distribution for the distance parameter $x$. As another special case with practical importance, the scenario in which the VLC receiver is uniformly distributed on the floor (ground) can be considered. In that case, a two dimensional uniform distribution can be employed over the area where the VLC receiver can 


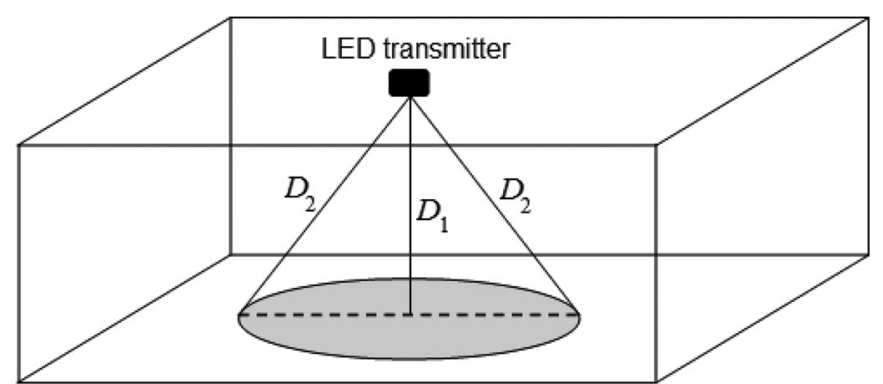

Fig. 5. The scenario in which the VLC receiver is located in the gray circular area according to a uniform distribution.

communicate with the LED transmitter. Let $\mathcal{A}_{v}$ denote this area. Based on the minimum and the maximum possible distances, which are denoted by $D_{1}$ and $D_{2}$, respectively (see Section III) and the fact that the LED transmitter and the VLC receiver are pointing in vertical directions, area $\mathcal{A}_{v}$ can be represented by a circle with a radius of $\sqrt{D_{2}^{2}-D_{1}^{2}}$, the center of which is located at the projection of the LED transmitter to the floor (please see Fig. 5 for an illustration). ${ }^{3}$ The use of such a circular area can be justified by the field of view of the VLC receiver, which imposes an upper limit on the incidence angle $\theta$ (see (3)) for communication between the LED transmitter and the VLC receiver [22]. When the position of the VLC receiver is uniformly distributed over the circular area $\mathcal{A}_{v}$, it can be shown, via some manipulation of random variables, that the distance $x$ between the LED transmitter and the VLC receiver is characterized by the following prior PDF:

$$
w(x)= \begin{cases}2 x /\left(D_{2}^{2}-D_{1}^{2}\right), & \text { if } D_{1} \leq x \leq D_{2} \\ 0, & \text { otherwise. }\end{cases}
$$

The ZZB bound can easily be evaluated for the prior PDF in (41) by inserting it into (8), (17), (20), and (22). Similarly, the ECRB expressions can be specified based on (27) and (41), which leads to similar expressions to those in (28)-(30). (In fact, the use of the prior PDF in (41) instead of the uniform PDF mainly increases the degree of $x$ in the numerator of the integral in (28); hence, the derivations stay almost the same.) In a similar fashion, the BCRB and the WCRB in Section V can also be evaluated for (8). Hence, specific expressions for the bounds can be obtained for the prior PDF in (41), as well.

In practical systems, due to synchronization errors and finite resolution of time delay estimates, the relation in (2) may not hold exactly. In order to derive the ZZB in the presence of such effects, (2) can be updated as $\tau=x / c+\varepsilon$, where $\varepsilon$ has a PDF denoted by $p_{\varepsilon}(\cdot)$. Then, from (1), the likelihood function can be obtained as (cf. (12))

$$
p(r(t) \mid x)=e^{k} \int p_{\varepsilon}(\epsilon) e^{-\frac{1}{2 \sigma^{2}} \int_{T_{1}}^{T_{2}}\left(r(t)-\frac{R_{p} \gamma}{x^{m}+3} s\left(t-\frac{x}{c}-\epsilon\right)\right)^{2} d t} d \epsilon .
$$

\footnotetext{
${ }^{3}$ In this case, $D_{1}$ corresponds to the height of the LED transmitter relative to the VLC receiver, which is also denoted by $h$ (see (4)).
}

Based on (42), the decision rule in (11) can be expressed, after some manipulation, as follows:

$$
\begin{aligned}
& \log \left(\int p_{\varepsilon}(\epsilon) e^{\frac{R_{p} \gamma \tilde{C}_{r s}(\vartheta+\delta, \epsilon)}{\sigma^{2}(\vartheta+\delta)^{m}+3}} d \epsilon\right)-\log \left(\int p_{\varepsilon}(\epsilon) e^{\frac{R_{p \gamma} \tilde{C}_{r s}(\vartheta, \epsilon)}{\sigma^{2} \vartheta}} d \epsilon\right) \\
& \stackrel{\mathcal{H}_{0}+3}{\risingdotseq} \frac{R_{p}^{2} \gamma^{2} E_{s}}{2 \sigma^{2}}\left(\frac{1}{(\vartheta+\delta)^{2 m+6}}-\frac{1}{\vartheta^{2 m+6}}\right)+\log \left(\frac{w(\vartheta)}{w(\vartheta+\delta)}\right)
\end{aligned}
$$

where $\tilde{C}_{r s}(x, \epsilon) \triangleq \int_{T_{1}}^{T_{2}} r(t) s(t-x / c-\epsilon) d t$. Since it is difficult to specify the PDF of the decision statistics in (43), a closed-form expression for $\mathrm{P}_{\min }$ in (23) may not be obtained. However, a Monte-Carlo approach can be adopted to evaluate $\mathrm{P}_{\mathrm{min}}$ based on the decision rule in (43). Then, the ZZB can be calculated numerically via (8).

As future work, theoretical limits for synchronous VLP systems can be considered for three-dimensional scenarios (i.e., when the height of the VLC receiver is unknown). In that case, the extended ZZB for vector parameter estimation [27] should be employed.

\section{REFERENCES}

[1] H. Burchardt, N. Serafimovski, D. Tsonev, S. Videv, and H. Haas, "VLC Beyond point-to-point communication," IEEE Commun. Mag., vol. 52, no. 7, pp. 98-105, Jul. 2014.

[2] P. Pathak, X. Feng, P. Hu, and P. Mohapatra, "Visible light communication, networking, and sensing: A survey, potential and challenges," IEEE Commun. Surveys Tuts., vol. 17, no. 4, pp. 2047-2077, Fourth Quarter 2015.

[3] S. Rajagopal, R. Roberts, and S.-K. Lim, "IEEE 802.15.7 visible light communication: Modulation schemes and dimming support," IEEE COmmun. Mag., vol. 50, no. 3, pp. 72-82, Mar. 2012.

[4] J. Armstrong, Y. Sekercioglu, and A. Neild, "Visible light positioning: A roadmap for international standardization," IEEE Commun. Mag., vol. 51, no. 12, pp. 68-73, Dec. 2013.

[5] T. Wang, Y. Sekercioglu, A. Neild, and J. Armstrong, "Position accuracy of time-of-arrival based ranging using visible light with application in indoor localization systems," J. Lightw. Technol., vol. 31, no. 20, pp. 3302-3308, Oct. 2013.

[6] X. Zhang, J. Duan, Y. Fu, and A. Shi, "Theoretical accuracy analysis of indoor visible light communication positioning system based on received signal strength indicator," J. Lightw. Technol., vol. 32, no. 21, pp. 4180-4186, Nov. 2014.

[7] L. Li, P. Hu, C. Peng, G. Shen, and F. Zhao, "Epsilon: A visible light based positioning system," in Proc. 11th USENIX Symp. Netw. Syst. Design Implementation, Seattle, WA, USA, Apr. 2014, pp. 331-343.

[8] D. Ganti, W. Zhang, and M. Kavehrad, "VLC-based indoor positioning system with tracking capability using Kalman and particle filters," in Proc. IEEE Int. Conf. Consum. Electron., Jan. 2014, pp. 476-477.

[9] Y. Eroglu, I. Guvenc, N. Pala, and M. Yuksel, "AOA-based localization and tracking in multi-element VLC systems," in Proc. 16th IEEE Annu. Wireless Microw. Technol. Conf., Apr. 2015, pp. 1-5.

[10] A. Jovicic, J. Li, and T. Richardson, "Visible light communication: Opportunities, challenges and the path to market," IEEE Commun. Mag., vol. 51, no. 12, pp. 26-32, Dec. 2013.

[11] S.-H. Yang, E.-M. Jung, and S.-K. Han, "Indoor location estimation based on LED visible light communication using multiple optical receivers," IEEE Commun. Lett., vol. 17, no. 9, pp. 1834-1837, Sep. 2013.

[12] S.-Y. Jung, S. Hann, and C.-S. Park, "TDOA-based optical wireless indoor localization using LED ceiling lamps," IEEE Trans. Consum. Electron., vol. 57, no. 4, pp. 1592-1597, Nov. 2011.

[13] W. Zhang, M. I. S. Chowdhury, and M. Kavehrad, "Asynchronous indoor positioning system based on visible light communications," Opt. Eng., vol. 53, no. 4, 2014, Art. no. 045105.

[14] Z. Zhou, M. Kavehrad, and P. Deng, "Indoor positioning algorithm using light-emitting diode visible light communications," Opt. Eng., vol. 51, no. 8, 2012, Art. no. 085009. 
[15] H.-S. Kim, D.-R. Kim, S.-H. Yang, Y.-H. Son, and S.-K. Han, "An indoor visible light communication positioning system using a RF carrier allocation technique," J. Lightw. Technol., vol. 31, no. 1, pp. 134-144, Jan. 2013.

[16] M. F. Keskin and S. Gezici, "Comparative theoretical analysis of distance estimation in visible light positioning systems," J. Lightw. Technol., vol. 34, no. 3, pp. 854-865, Feb. 2016.

[17] M. Bilgi, A. Sevincer, M. Yuksel, and N. Pala, "Optical wireless localization," Wireless Netw., vol. 18, no. 2, pp. 215-226, Feb. 2012.

[18] S.-H. Yang, H.-S. Kim, Y.-H. Son, and S.-K. Han, "Three-dimensional visible light indoor localization using AOA and RSS with multiple optical receivers," J. Lightw. Technol., vol. 32, no. 14, pp. 2480-2485, Jul. 2014.

[19] A. Sahin, Y. S. Eroglu, I. Guvenc, N. Pala, and M. Yuksel, "Hybrid 3D localization for visible light communication systems," J. Lightw. Technol., vol. 33, no. 22, pp. 4589-4599, Nov. 2015.

[20] D. Dardari, C.-C. Chong, and M. Win, "Improved lower bounds on timeof-arrival estimation error in realistic UWB channels," in IEEE Int. Conf. Ultra-Wideband, Sep. 2006, pp. 531-537.

[21] H. L. Van Trees, and K. L. Bell, Eds., Bayesian Bounds for Parameter Estimation and Nonlinear Filtering/Tracking. Hoboken, NJ, USA: Wiley, 2007.

[22] E. Gonendik and S. Gezici, "Fundamental limits on RSS based range estimation in visible light positioning systems," IEEE Commun. Lett., vol. 19, no. 12, pp. 2138-2141, Dec. 2015.

[23] Z. Liu, J. Shen, Z. Yao, M. Lu, and Y. Zhao, "The Ziv-Zakai bound for time-of-arrival estimation of new generation GNSS signals," in Proc. Int. Tech. Meeting Inst. Navig., Monterey, CA, USA, Jan. 2016, pp. 639-646.

[24] D. Dardari and M. Win, "Ziv-Zakai bound on time-of-arrival estimation with statistical channel knowledge at the receiver," in Proc. IEEE Int Conf. Ultra-Wideband, Sep. 2009, pp. 624-629.

[25] H. V. Poor, An Introduction to Signal Detection and Estimation. New York, NY, USA: Springer, 1994.

[26] Y. Qi and H. Kobayashi, "Cramér-Rao lower bound for geolocation in non-line-of-sight environment," in Proc. IEEE Int. Conf. Acoust., Speech, Signal Process., May 2002, vol. 3, pp. III-2473-III-2476.

[27] K. L. Bell, Y. Steinberg, Y. Ephraim, and H. L. V. Trees, "Extended ZivZakai lower bound for vector parameter estimation," IEEE Trans. Inf. Theory, vol. 43, no. 2, pp. 624-637, Mar. 1997.
Musa Furkan Keskin received the B.S. and M.S. degrees, in 2010 and 2012, respectively, from the Department of Electrical and Electronics Engineering, Bilkent University, Ankara, Turkey, where he is currently working toward the $\mathrm{Ph} . \mathrm{D}$. degree. His main research interests include statistical signal processing, wireless communications, and visible light positioning.

Erdal Gonendik received the B.S. degree from the Department of Electrical and Electronics Engineering, Middle East Technical University, Ankara, Turkey, and the M.S. degree from Bilkent University, Ankara, where he is currently working toward the Ph.D. degree at the Department of Electrical and Electronics Engineering. His main research interests include statistical signal processing, wireless communications, and visible light positioning.

Sinan Gezici received the B.S. degree from Bilkent University, Ankara, Turkey in 2001, and the Ph.D. degree in electrical engineering from Princeton University, Princeton, NJ, USA, in 2006. From 2006 to 2007, he worked at Mitsubishi Electric Research Laboratories, Cambridge, MA, USA. Since 2007, he has been with the Department of Electrical and Electronics Engineering, Bilkent University, where he is currently an Associate Professor.

His research interests include detection and estimation theory, wireless communications, and localization systems. Among his publications in these areas is the book entitled Ultra-Wideband Positioning Systems: Theoretical Limits, Ranging Algorithms, and Protocols (Cambridge, U.K.: Cambridge Univ. Press, 2008). He is an Associate Editor for the IEEE TRANSACTIONS ON COMMUNICATIONS, IEEE WIRELESS COMMUNICATIONS LETTERS, and Journal of Communications and Networks. 